\title{
Théologiques
}

\section{Troisième Colloque de la revue}

\section{Marie-Andrée Bertrand}

Volume 4, numéro 1, mars 1996

Les Rites : céder en résistant

URI : https://id.erudit.org/iderudit/602436ar

DOI : https://doi.org/10.7202/602436ar

Aller au sommaire du numéro

Éditeur(s)

Faculté de théologie de l'Université de Montréal

ISSN

1188-7109 (imprimé)

1492-1413 (numérique)

Découvrir la revue

Citer ce document

Bertrand, M.-A. (1996). Troisième Colloque de la revue. Théologiques, 4(1), 135-138. https://doi.org/10.7202/602436ar d'utilisation que vous pouvez consulter en ligne.

https://apropos.erudit.org/fr/usagers/politique-dutilisation/ 
Théologiques 4/1 (1996) 135-138.

\title{
Troisième Colloque de la revue
}

\author{
Marie-Andrée BERTRAND \\ Département de criminologie \\ Université de Montréal
}

Depuis plus d'un an déjà, à la suite de la parution de chaque numéro de la revue Théologiques, se tient un Colloque qui reprend, en les prolongeant, les réflexions produites sur la thématique traitée. Le 20 novembre dernier, avait lieu le troisième Colloque de la revue dont le thème était : "Symbolique urbaine et foi chrétienne ". Outre les interventions de Benôt Bégin, André Charron et Myriam El Yamani, on avait demandé à Mme Marie-Andrée Bertrand de donner une première réaction à sa lecture de l'ensemble du numéro. Nous reproduisons ici, en écho à ce Colloque, l'intervention de Mme Bertrand. (G. L.)

Le titre de ce numéro thématique annonçait deux pôles, la ville et la foi chrétienne. Des deux c'est le premier qui occupe le plus d'espace et revêt le plus d'importance dans la livraison: le thème de la ville est abordé dans neuf contriburions et traité avec générosité dans l'éclairage de diverses disciplines; celui de la foi chrétienne est l'objet central des articles de deux auteurs et il est analysé dans ses rapports conflictuels avec la ville dans deux autres contributions.

La ville contemporaine est surtout décrite dans ce numéro comme l'ennemie des rapports humains personnalisés, communautaires et responsables, comme un lieu de conflit entre personnes et institutions ${ }^{1}$. $\grave{A}$ titre d'exemples de ce procès, voir par exemple Greinacher qui rappelle que la ville est un "Moloch dévorant" (page 21); Wittner et Welzer-Lang qui croient qu'il faut la "casser,... (la) changer» (40). Pour Myriam El Yamani, "la ville ne parvient pas vraiment à reconstituer un tissu social

1 Deux des auteurs, Charron et El Yamani soulignent à l'occasion la richesse et la variété culturelles de la grande ville sans cependant s'éloigner, dans l'ensemble, du diagnostic négatif des autres collaborateurs. 
cohérent », victime qu'elle est de la mondialisation des marchés, des flux migratoires (46). André Charron estime que «(l')urbain et les composantes de la modernité et de l'industrialisation provoquent l'affaiblissement des communautés d'appartenance. (...) On parle même d'éclatement des communautés d'appartenance " (66-67). On y vit "l'exclusion", écrit-il. L'exclusion est le thème qui domine le propos de Wittner et Welzer-Lang. Pour Bégin, la ville contemporaine condamne ses résidents à la mort lente de leur rôle de ciroyens (97), elle les réduit au rôle de payeurs de taxes. Lamoureux et Myre eux-mêmes, bien qu'occupés à traiter en substance d'autres sujets, consacrent chacun un paragraphe (p. 114 et 116 respectivement) à décrire de façon saisissante, voire dramatique, les problèmes que la ville contemporaine engendre.

Ce procès de la ville contemporaine utilise les termes d'une critique plus large, celle de la modernité et de la crise des institutions. La modernité devient le moment par excellence d'une crise de la foi et de la pratique religieuses; la ville, le lieu privilégié de leur désintégration. L'époque moderne et la ville sont les boucs émissaires des malheurs de la foi.

La critique de la modernité à laquelle emprunte largement ce numéro est un thème important traité par un nombre impressionnant de sociologues et de philosophes. La moitié de ces derniers - que j'appellerai ou qui se nomment eux-mêmes précisément "les critiques de la modernité " - estiment que nous vivons une "modernité avancée ${ }^{2}$ » qui n'a déjà plus grand chose à voir avec la période qui a pris naissance à l'époque des Lumières $^{3}$. Pour plusieurs de ces "critiques de la modernité ", il est possible et désirable d'en sauver les acquis. Revenant à Théologiques, je dirai que les contributions nous présentent la ville contemporaine qui surfe sur cette scène de "high modernity ", portée par la vague de la société marchande, du libéralisme économique et des progrès technologiques euxmêmes en pleine implosion.

2 L'expression "late modernity " est de Anthony Giddens. Voir note 3 pour la référence.

3 Voir par exemple J. HABERMAS, Le discours philosophique de la modernité. Paris, Gallimard, 1988. Voir aussi du même auteur, Raison et légitimité : problèmes de légitimation dans le capitalisme avancé. Paris, Payot, 1978. Également, Allan Bloom, L'àme désarmée. Paris, Julliard, 1987. Anthony GIDDENS, Modernity and Self-Identity. Self and Society in the Late Modern Age. Stanford, CA, Stanford University Press, 1991, ou encore: The Consequences of Modernity. Cambridge, Polity Press, 1990. Alain TOURAINE, Critique de la modernité. Paris, Arthème Fayard, 1992. 
À cet égard, le numéro de Théologiques nous offre une analyse importante de la ville contemporaine, de son histoire, de ses rapports avec la religion et la foi, sur fond de critique de la modernité.

J'aurais aimé pour ma part qu'on nous parle aussi de l'avenir; de l'avenir de la ville et de la pratique chrétienne. Un avenir à chercher non pas dans une boule de cristal mais, pour partie, chez les analystes de la scène sociale, économique, politique, religieuse, et d'autre part dans notre propre expérience de la ville d'ici ${ }^{4}$. Il s'agirait alors de voir venir, par exemple, l'accroissement ou la régression des phénomènes constatés tels l'exclusion sociale, le fractionnement des anciens groupes d'appartenance, - et de dessiner des pratiques sans doute nouvelles, comme le fait Lamoureux.

Mais si, au terme de notre analyse, rien dans l'évolution ainsi entrevue ne vient ni ne peut, à moyen terme, contrer l'exclusion et le fractionnement, que faire puisque la pastorale ne semble pas pouvoir s'accommoder de l'individualisme contemporain? - Cette hypothèse, qui apparaît pessima à plusieurs, n'est pas dénuée de fondement si l'on en croit plusieurs théoriciens éminemment, tout aussi nombreux que les critiques de la modernité cités précédemment. Pour les postmodernes, en effet, nous sommes déjà dans une autre ère qui ne peut plus fonder ses manières de penser sur ce qui nous a portés jusqu'à maintenant. Les récits ${ }^{5}$ que sont la Bible, les Lumières et la Raison, la lutte des classes, la foi dans le Progrès et la Science nous ont montré leurs limites; il s'agit maintenant de chercher des voies modestes pour la connaissance et l'action, des chemins sans prétention aux réponses universelles, respectueux des différences. Ainsi, alors que les critiques de la modernité espèrent re-fonder des pratiques anciennes, universelles, et donner toute leur chance à la Raison et à la rationalité qui «n'ont pas fini de donner » selon Touraine ${ }^{6}$,

4 Un « ici » à ne jamais confondre comme certains auteurs dans ce numéro ont été tentés de le faire avec la "scène » urbaine en France, et surtout à bien distinguer du Moyen-Orient et des pays du sud où ville et modernité éclatent bien différemment.

5 Voir notamment J. F. LYOTARD, La condition postmodeme. Paris, Minuit, 1979. Voir aussi Moralités postmodernes. Paris, Gallimard, 1983 et 1993. Du même auteur, voir aussi Le crépuscule du devoir, l'éthique indolore des nouveaux temps démocratiques. Paris, Gallimard, 1992. Umberto ECO, Apostille à Le nom de la rose. Paris, Grasset, 1982. Richard RORTY, Science et solidarité, la vérité sans le pouvoir. Paris, Éditions de l'Éclat, 1990.

6 Voir note, 3, TOURAINE, Critique de la modemité. 
les seconds croient que les fondements anciens ne leur offrent plus d'assises et tentent de repenser radicalement connaissances et pratiques.

Dans l'ensemble, les auteurs de ce numéro sont plutôt du côté de Touraine : au plan de la praxis, la majorité croit possible ou impératif de sauver les "acquis " de la modernité, de la rationalité et des récits, tandis que Lamoureux témoigne d'une expérience qui s'accommode de l'éclatement des "anciens" liens et rapports sociaux.

On aurait aimé que les premiers, les renants d'une reconstruction de la modernité, s'expliquent de ce qui les fait opter pour cette hypothèse plutôt que pour celle de Lyotard, Lipovetsky, Rorty, etc. Le fait même de considérer la seconde hypothèse infléchir les pratiques. 